\title{
RANDOMIZED APPROACH TO DETERMINE DYNAMIC STRENGTH OF ICE
}

Nikolay Granichin

Theory of elasticity department

Saint Petersburg State University Russia

kolya30.87@yandex.ru
Grigory Volkov

Theory of elasticity department

Saint Petersburg State University

Russia

Institute for Problems

in Mechanical Engineering

Saint Petersburg, Russia

g.volkov@spbu.ru

\author{
Marina Volkova \\ Mathematical theory \\ of decision making \\ in economy department \\ Saint Petersburg State University \\ Russia \\ m.volkova@spbu.ru
}

\author{
Yuri Petrov \\ Theory of elasticity department \\ Saint Petersburg State University \\ Russia \\ Institute for Problems \\ in Mechanical Engineering \\ Saint Petersburg, Russia \\ y.v.petrov@spbu.ru
}

Article history:

Received 03.11.2021, Accepted 25.11.2021

\begin{abstract}
The randomized method of Sign-Perturbed Sums (SPS) is applied within the framework of the incubation time approach to evaluate the dynamic strength of ice. The experimental data of [Carney et al., 2006; Wu and Prakash, 2015; Saletti et al., 2019] is analysed in order to estimate strength parameters of ice and describe the observed strain-rate sensitivity curves. The independence of incubation time value on the ice temperature is established in contrast with the significant dependency of the critical stress parameter. The obtained confidence interval of the spalled ice is in good correspondence with the scatter observed experimentally.
\end{abstract}

\section{Key words}

Sign-Perturbed Sums, incubation time, dynamic strength of ice

\section{Introduction}

Estimation of materials strength properties is an important daily task of modern industry. One of the materials in the field of interest is ice. The ice fields interact with arctic ships and platforms working to the oil and gas extraction. Thus, the problem of ice strength properties becomes critical since such interactions can damage a vessel or platform. A number of various tests were conducted under quasi-static and dynamic loading for wide range of temperatures by $\mathrm{Wu}$ and Prakash [Wu and Prakash, 2015]. They established that the accepted critical stress criterion does not provide proper results for dynamic cases as, in dynamics, the ultimate stresses strongly depend on the load rate.

In addition, the issue of studying the ice characteristics under high-speed loads is important not only for vessels travelling through the Arctic seas but also for spaceships [Carney et al., 2006]. During the atmospheric flight, spaceships can collide with various types of debris and it is necessary for the spacecraft body to withstand these impacts. It is believed that dense objects do not pose any danger, because the relative impact density is moderate, however, objects with low density slow down quickly and during the collision, the speed is quite high. Ice slows down fast enough to consider an impact as a potential problem, so it is necessary to have more knowledge about the strength characteristics of ice under highspeed impact.

It is also important that the ice fracture mechanism can be different in dynamics and statics. The yield deformation is typical to quasi-static loading, whereas the brittle fracture prevails under high rate intensive impacts [Schulson, 2001; Schulson and Buck, 1995]. This peculiarity of internal ice structure can be the reason that 
conventional approaches based on the concept of critical stress wrongly predict the strength in dynamics.

Recently, a number of tests were conducted on different types of ice under various temperatures in order to determine the dependence of the ice strength on strain rate [Wu and Prakash, 2015; Carney et al., 2006; Dempsey et al., 1999; Saletti et al., 2019; Yasui et al., 2017; Combescure et al., 2011]. This study is devoted to the analysis of mentioned experimental data within the framework of the structural-temporal approach based on the incubation time criterion. The main idea of the criterion is that every transient process like fracture does not happen instantly, and the material's parameter, the incubation time, determines the characteristic time of the process. Thus, the material strength in dynamics is stipulated by this parameter. This method has recommended itself as an effective tool for the solution of different problems related to prediction ultimate stress level under intensive high-rate loading[Petrov, 2004; Volkov et al., 2021]. The main issue is estimating the incubation time value through the experimental data of dynamic tests. The randomized Sign-Perturbed Sums (SPS) method [Csáji et al., 2014] is applied in this study since it has weak restrictions on model function and almost no assumption of the random noise distribution. Originally, SPS was formulated for linear model function, whereas the experimental regularity of ultimate strength on load rate is definitely nonlinear. It was shown that this randomized method could be applied to solve dynamic fracture problems, and the model function obtained within the incubation time approach obeys all necessary conditions of SPS[Volkova et al., 2017; Volkova et al., 2018].

The paper is organized as follows. In Section 1, the formal problem of estimation dynamic strength properties of the ice is discussed. Section 2 introduces the general problem settings, and it consists of two parts that describe basic ideas and concepts of the incubation time approach and Sign-Perturbed Sums methods. The results of experimental data analysis are considered in Section 3. Section 4 concludes the paper.

\section{Problem Statement}

All test results can be considered in accordance with the following observation scheme

$$
y_{i}=\varphi\left(\vartheta, u_{i}\right)+v_{i}, \quad i=1,2, \ldots, N
$$

where $y_{i}$ is observed value of $i$-th test, $\varphi\left(\vartheta, u_{i}\right)$ is a model function with a control input parameter $u_{i}$ and an internal parameter $\vartheta$, which needs to be estimated through the tests, $v_{i}$ is a random noise. The scheme (1) is accepted in further analysis of material dynamic strength within the incubation time approach. Thus, $y_{i}$ is the experimentally observed value of ultimate stress level $\sigma_{*}$, $\vartheta$ corresponds to the incubation time of material $\tau$ and $u_{i}$ can be associated to the strain rate of loading $\dot{\varepsilon}_{i}$. The model function $\varphi\left(\tau, \dot{\varepsilon}_{i}\right)$ is also should be determined by the structural temporal approach.

It should be noted, that the number of dynamic tests $N$ often is not large enough to apply conventional statistical analysis in order to consider the noise influence. Therefore, the randomized method of Sign-Perturbed Sums is chosen as a basic tool for this problem.

\subsection{Incubation Time Approach}

The incubation time approach was applied to describe the ice strength in this study. The general form of the criterion can be represented by

$$
\frac{1}{\tau} \int_{t-\tau}^{t}\left(\frac{\sigma\left(t^{\prime}\right)}{\sigma_{c}}\right)^{\alpha} d t^{\prime} \leq 1
$$

where $\sigma_{c}$ is critical stress, $\tau$ is incubation time of fracture and $\alpha$ is dimensionless parameter. In further analysis, it is assumed that $\alpha=1$ since it is typical for a wide range of materials. The function $\sigma\left(t^{\prime}\right)$ describes the time profile of loading stresses. According to (2) the fracture manifestation corresponds to the equality for the some time moment $t_{*}$. Thus, the criterion (2) permits the calculation of a dependence of the fracture time on the threshold value of some parameters of a specific load function.

The experimental tests to be analyzed were performed on a Split Hopkinson's Pressure Bars (SHPB) equipment. Usually, these experimental scheme provides linear growth of loading stresses, and therefore the load function can be set as

$$
\sigma(t)=h(t) k \dot{\varepsilon} t,
$$

where $h(t)$ is Heaviside step function and $k$ is the material Young modulus. Substitution the function (3) to criterion (2) leads to following equation in unknown fracture time $t_{*}$

$$
h\left(t_{*}\right)\left(\frac{t_{*}}{\tau}\right)^{2}-h\left(t_{*}-\tau\right)\left(\frac{t_{*}}{\tau}-1\right)^{2}=s
$$

where $s=2 \sigma_{c} /(k \dot{\varepsilon} \tau)$ is dimensionless parameter, which value depends on strain-rate of load impacts. Fracture time $t_{*}$ is positive value, hence $h\left(t_{*}\right)=1$ and there are two cases: when $t_{*}>\tau$ or $t_{*} \leq \tau$. From expression (4) it follows that $s=1$ for $t_{*}=\tau$, therefore the equation determining the fracture moment $t_{*}$ is

$$
\left\{\begin{array}{lr}
\left(t_{*} / \tau\right)^{2}=s, & s<1 \\
\left(t_{*} / \tau\right)^{2}-\left(t_{*} / \tau-1\right)^{2}=s, & s \geq 1 .
\end{array}\right.
$$

The substitution of the roots of the equation (5) into the load function (3) leads to the definition of the model function $\varphi\left(\tau, \dot{\varepsilon}_{i}\right)$

$$
\sigma_{*}(\dot{\varepsilon})=\varphi(\tau, \dot{\varepsilon})=\left\{\begin{array}{l}
\sigma_{c}+\frac{\tau}{2} k \dot{\varepsilon}, \dot{\varepsilon} \leq 2 \sigma_{c} / k \tau \\
\sqrt{2 \sigma_{c} \tau k \dot{\varepsilon}}, \dot{\varepsilon}>2 \sigma_{c} / k \tau
\end{array}\right.
$$




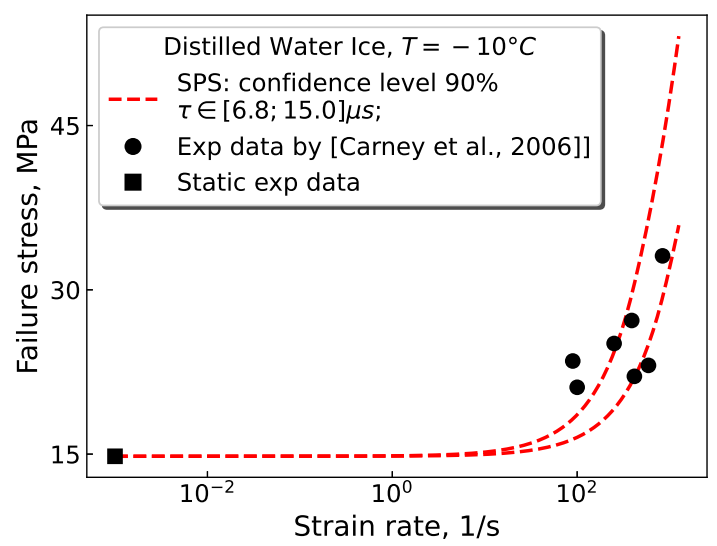

Figure 1. Dependence of the failure stress on the strain-rate in comparison to experimental data for the distilled water ice [Carney et al., 2006]. Model parameters are $\tau \in[6.8 ; 15.0] \mu \mathrm{s}, \sigma_{c}=14.8 \mathrm{MPa}$

Therefore, the incubation time approach allows describing strain-rate dependence of ultimate fracture stresses under dynamic loading. The main task is to estimate the model parameter $\tau$ for certain materials by analysing real experimental data.

\subsection{Sign-Perturbed Sums Method}

The Sign-Perturbed Sums approach provides the estimation of the goal parameter in the form of some confidence interval that contains the actual value of the parameter with a given probability. It was proven in [Volkova et al., 2018] that the nonlinear function $\varphi(\tau, \dot{\varepsilon})$ obeys necessary conditions of applicability of the SPS method. The only additional assumption is that the random noise has to be symmetrical. This is quite reasonable due to the consideration that the performance of the experiments was high enough to avoid all bias errors with a non zero mean value.

The implementation of SPS to the task mentioned above can be realized in two steps. At first, some number of trial values of the goal parameter $\tau$ should be set as some interval. Then the $S P S-\operatorname{Indicator}(\tau)$ SPSIndicator procedure checks every value from the trial ones whether the confidence set includes it or not. The main concept of SPS is based on the sign perturbation of residual error for a model curve value and experimental data. For the true value of the model parameter, the sum of perturbed residuals should not be different to their sum with original signs. The main steps of the $S P S$ - Indicator $(\tau)$ procedure are as follows.

1. For the given $\tau$ compute the prediction error: $\delta_{t}(\tau)=\sigma_{t}^{*}-\varphi\left(\tau, \dot{\varepsilon}_{t}\right), \quad t=1,2, \ldots, T$, where $\left(\sigma_{t}^{*}, \dot{\varepsilon}_{t}\right)$ are observed data of experiment.

2. Evaluate

$$
\begin{array}{r}
H_{0}(\tau)=\sum_{t=1}^{T} \varphi_{\tau}^{\prime}\left(\dot{\varepsilon}_{t}, \tau\right) \delta_{t}(\tau) \\
H_{i}(\tau)=\sum_{t=1}^{T} \beta_{i, t} \varphi_{\tau}^{\prime}\left(\dot{\varepsilon}_{t}, \tau\right) \delta_{t}(\tau)
\end{array}
$$

for $i=1,2, \ldots, M-1$.

3. Order $\left\|H_{i}(\tau)\right\|$ from smallest to biggest one. Place equal sums randomly in accordance with the uniform distribution into the corresponding to their value position in the ordering.

4. Compute the $\operatorname{rank} \mathcal{R}(\tau)$ as a number of $\left\|H_{0}(\tau)\right\|$ in the ordering.

5. Return 1 if $\mathcal{R}(\tau) \leq M-q$, otherwise Return 0 .

Thus, the confidence interval has the form

$$
\widehat{T}=\{\tau \in \mathbb{R} \mid \operatorname{SPS}-\operatorname{indicator}(\tau)=1\} .
$$

The main steps of the SPS - Indicator $(\tau)$ procedure are as Integer parameters $M$ and $q$ should be chosen in advance in order to set the confidence level of the estimation as a probability of the resulting interval $\widehat{T}$ contains the actual value of the incubation time $\tau^{*}$.

$$
\operatorname{Prob}\left(\left\{\tau^{*} \in \widehat{T}\right)=1-\frac{q}{M} .\right.
$$

It should be noted, that SPS is a randomized method and it can provide results slightly different one another for the same initial data. Parameter $M$ is the number of sets of random signs $\beta_{i}, t$, so there is a reasonable assumption that the results with an equal confidence level are more stable for a greater value of $M$.

\section{Analysis and Results}

The method of data analysis considered above is applied to experimental results obtained by Schulson in [Carney et al., 2006]. The results of modelling are demonstrated in Figure 1. The $90 \%$ confidence interval for $\tau$ is $[6.8 ; 15.0] \mu s$ and modelling curves describe adequately the strain rate sensitivity of the ultimate stresses and the experimental scatter of dynamic branch of the strength.

A number of test under various temperatures were provided by Wu and Prakach in [Wu and Prakash, 2015]. They observed that in statics, the critical stress grows with the temperature decrease. These data were also analysed by the developed method, and confidence intervals were obtained for each temperature. The results are collected in Table1. All confidence intervals seem to be close to each other; therefore, it can indicate that the dynamic strength of the materials almost does not change with the temperature drop and the actual value of $\tau \in[7.3 ; 13.7] \mu s$ The data for all temperatures were plotted in the single graph of the dimensionless strength versus strain rate (Figure 2) in order to emphasise that the strain rate sensitivity is stable concerning the ice temperature. These data were also analysed in order to obtain intervals of $80 \%$ confidence level. These intervals 


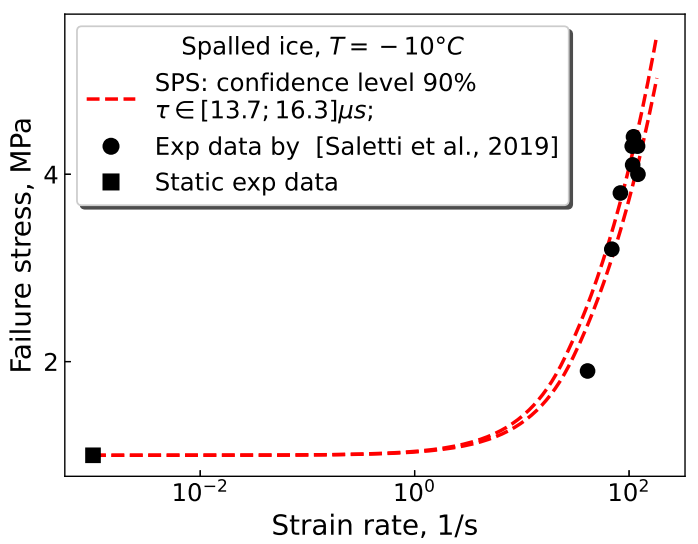

Figure 3. Dependence of the failure stress on the strain rate in comparison to experimental data for the spalled ice [Saletti et al., 2019]. Model parameters are $\tau \in[13.7 ; 16.3] \mu \mathrm{s}, \sigma_{c}=1.0 \mathrm{MPa}$

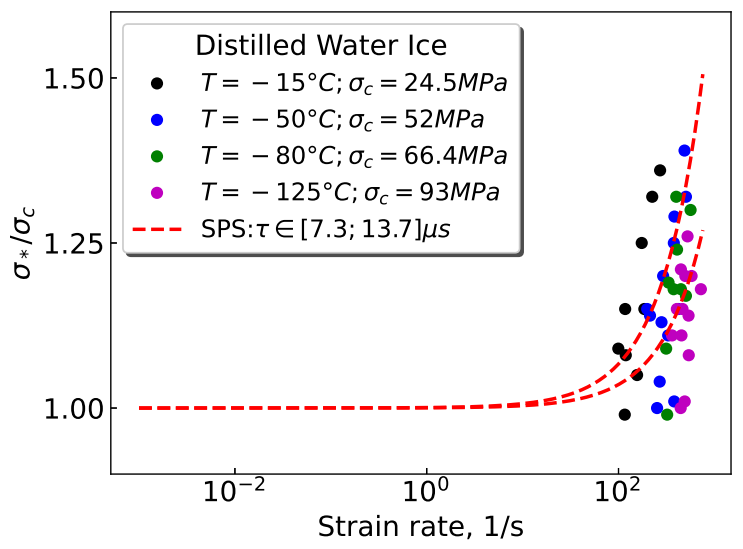

Figure 2. Dependence of the normalised failure stress on the strain rate in comparison to experimental data for the distilled water ice [Wu and Prakash, 2015]. Model parameters are $\tau \in[7.3 ; 13.7] \mu \mathrm{s}$, $\sigma_{c}=52.0 \mathrm{MPa}$

Table 1. Confidence level is $90 \%$

\begin{tabular}{c|c|c|c}
\hline$T,{ }^{\circ} C$ & $\tau_{\min }, \mu s$ & $\tau_{\max }, \mu s$ & $\tau_{\text {avg }}, \mu s$ \\
\hline \hline-125 & 7.5 & 12.9 & 10.2 \\
-80 & 9.0 & 14.8 & 11.9 \\
-50 & 7.1 & 13.3 & 10.2 \\
-15 & 6.3 & 12.8 & 9.6 \\
-10 & 6.8 & 15.0 & 10.7 \\
\hline
\end{tabular}

became more bounded than $90 \%$ ones, but the average value occurred almost the same, about $10.5 \mu \mathrm{s}$

The spalled ice tests results ( [Saletti et al., 2019]) were also treated by the developed method. This experimental scheme suppose the specimen fracture under tensile stress state. Provided analysis shows that the incubation time is in the following range $\tau \in[13.7 ; 16.3] \mu \mathrm{s}$ (Figure 3 ), which is pretty close to the values of the compression case. It means that the strain rate sensitivity is almost the same for compression and tensile fracture processes, despite the critical stresses being pretty different.

The last experimental data were also supported by a given scatter of the strain rate. Figure 4 demonstrates the modelling curves and experimental points with the mentioned scatter in dynamic range. It can be noted that that the calculated band is in a good coincidence with the experimentally estimated range. Thus, the SPS method permits a description of the strain rate sensitivity of the material strength and the accuracy of the predicted model curve simultaneously.

\section{Conclusion}

A few sets of experimental data were analysed in order to estimate the dynamic strength properties of ice within the framework of the incubation time approach. The special method based on the randomized algorithm to calculate the actual value of the model parameter was developed. The Sign-Perturbed Sums approach allowed unified estimating of the incubation time parameter by dynamic data analysis. A few number of experimental series on dynamic compression fracture of ice were treated in order to obtain the actual values of the incubation time for various temperatures. This estimation in form of the confidence interval permitted to notice that the incubation time almost does not vary with the temperature. It means that dynamic strength properties are independent on thermal conditions, whereas the static

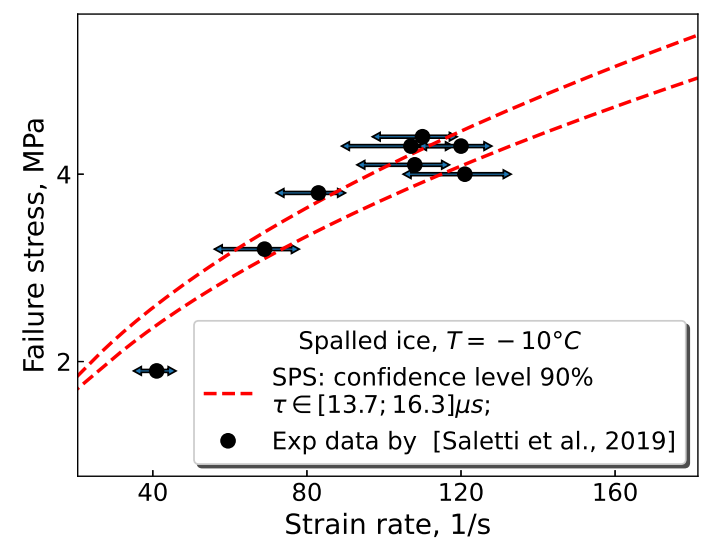

Figure 4. Dependence of the failure stress on the strain rate at linear scale in comparison to the data observed for the spalled ice [Saletti et al., 2019]. Arrows indicate the experimental scatter of the strain rate. Model parameters are $\tau \in[13.7 ; 16.3] \mu \mathrm{s}, \sigma_{c}=1.0 \mathrm{MPa}$ 
ones significantly change. This phenomenon is in accordance with the thermal fluctuation theory of the strength. The critical stress determines strength in statics and it is equilibrium characteristic. Thus it can be explained as a result of fluctuation processes in the internal material structure. Whereas in dynamics, there is not enough time to make the influence of fluctuations significant concerning dynamic strength properties of a material.

The developed method also provided good results for the tensile experiment data observed under the spall test. The estimation of the incubation time value recalled that the strain rate sensitivity under compression and tensile loading is almost the same. The obtained confidence interval for $\tau$ is also well corresponded to the experimentally observed scatter. It can be concluded that there is no need to evaluate the accuracy of the observed experimental data carefully since the suggested data treatment is able to predict the possible deviations of the ultimate stress.

\section{Acknowledgements}

The research is supported by RFBR projects \# 20-3170053 and \# 20-01-00291.

\section{References}

Carney, K. S., Benson, D. J., DuBois, P., and Lee, R. (2006). A phenomenological high strain rate model with failure for ice. International Journal of Solids and Structures, 43 (25-26), pp. 7820-7839.

Combescure, A., Chuzel-Marmot, Y., and Fabis, J. (2011). Experimental study of high-velocity impact and fracture of ice. International Journal of Solids and Structures, 48 (20), pp. 2779-2790.

Csáji, B. C., Campi, M. C., and Weyer, E. (2014). Signperturbed sums: A new system identification approach for constructing exact non-asymptotic confidence regions in linear regression models. IEEE Transactions on Signal Processing, 63 (1), pp. 169-181.

Dempsey, J., Adamson, R., and Mulmule, S. (1999). Scale effects on the in-situ tensile strength and frac- ture of ice. part ii: First-year sea ice at resolute, nwt. International journal of fracture, 95 (1), pp. 347-366.

Petrov, Y. V. (2004). Incubation time criterion and the pulsed strength of continua: fracture, cavitation, and electrical breakdown. In Doklady Physics, vol. 49, Springer, pp. 246-249.

Saletti, D., Georges, D., Gouy, V., Montagnat, M., and Forquin, P. (2019). A study of the mechanical response of polycrystalline ice subjected to dynamic tension loading using the spalling test technique. International Journal of Impact Engineering, 132, pp. 103315.

Schulson, E. and Buck, S. (1995). The ductile-to-brittle transition and ductile failure envelopes of orthotropic ice under biaxial compression. Acta metallurgica et materialia, 43 (10), pp. 3661-3668.

Schulson, E. M. (2001). Brittle failure of ice. Engineering fracture mechanics, 68 (17-18), pp. 1839-1887.

Volkov, G., Logachev, A., Granichin, N., Zhao, Y.-P., Zhang, Y., and Petrov, Y. (2021). The influence of background ultrasonic field on the strength of adhesive zones under dynamic impact loads. Materials, 14 (12), pp. 3188.

Volkova, M., Granichin, O., Volkov, G., and Petrov, Y. V. (2018). On the possibility of using the method of signperturbed sums for the processing of dynamic test data. Vestnik St. Petersburg University, Mathematics, 51 (1), pp. 23-30.

Volkova, M., Volkov, G., Granichin, O., and Petrov, Y. (2017). Sign-perturbed sums approach for data treatment of dynamic fracture tests. In 2017 IEEE 56th Annual Conference on Decision and Control (CDC), IEEE, pp. 1652-1656.

Wu, X. and Prakash, V. (2015). Dynamic compressive behavior of ice at cryogenic temperatures. Cold Regions Science and Technology, 118, pp. 1-13.

Yasui, M., Schulson, E. M., and Renshaw, C. E. (2017). Experimental studies on mechanical properties and ductile-to-brittle transition of ice-silica mixtures: Young's modulus, compressive strength, and fracture toughness. Journal of Geophysical Research: Solid Earth, 122 (8), pp. 6014-6030. 